\title{
The Classification of M 1-78
}

\author{
G. T. Gussie \\ Department of Physics \\ University of Tasmania \\ GPO Box 252C \\ Hobart, Tasmania \\ Australia, \\ 7001 \\ internet: Grant.Gussie@phys.utas.edu.au
}

Submitted: 28 June 1994

Resubmitted: 26 August 1994

Accepted: 14 September 1994 


\begin{abstract}
The published properties of M1-78 are discussed with the purpose to resolve the object's classification as either a planetary nebula or an ultracompact HII region. A classification as a planetary nebula is rejected primarily because of the high luminosity ofthe object, but because of the chemical composition and expansion velocity of the nebula, anovel classification is proposed instead: that of an ultracompact HII region with a post-main sequence central star (possibly a WN star). It must therefore follow that observableultracompact HII regions persist beyond the main sequence lifetimes of at least some massive stars, and so cannot be transient phenomena that are seen only during pre-main sequence or early mainsequence evolution.
\end{abstract}


1) Introduction

The nebula M1-78 is an interesting and enigmatic object which has been the subjectof optical, radio, and submillimetre observations for several decades.However its classification still remains undetermined. It has been classified as both anultracompact (hereafter UC) HII region and a planetary nebula (hereafter PN). The UC HII regions are believed to be the products of massive star formation, and result when hot young starsphoto-ionise their natal molecular gas clouds. A PN is thought to result when a moremoderately massed star reaches the end of its main-sequence and red giant life, ascends theasymptotic giant branch (hereafter AGB), and then sheds its atmosphere to surrounding space to reveal itshot core. Ultracompact $\mathrm{HII}$ regions and $\mathrm{PNe}$ are therefore very different in their respectiveorigins, but perhaps surprisingly they are not always easily distinguishableobservationally. This paper discusses their distinguishing characteristics and the evidence for theclassification of the object M 1-78.

\section{2) Evidence for the Classification of M1-78.}

One distinguishing characteristic between a planetary nebula and acompact HII region is that the mean dust temperature of $\mathrm{PNe}$ is considerably higher than themean dust temperature near UC HII regions. As a result of this, PNe and UC HII regions occupy different regions of a far-infrared colour-colour diagram. M1-78's far infrared colour indexes are

$$
\begin{aligned}
& \frac{F_{v}(12 \mu \mathrm{m})}{F_{v}(25 \mu \mathrm{m})}=0.22 \\
& \frac{F_{v}(25 \mu \mathrm{m})}{F_{v}(60 \mu \mathrm{m})}=0.30
\end{aligned}
$$

and therefore the nebula occupies a position on the infraredcolour-colour plot in the transition region between planetary nebulae and compact HII regions (figure 1). Thus neither classification is clearly indicated, although possibly the planetarynebula classification is weakly favoured.

Another distinguishing characteristic is that (as a whole) compactHII regions tend to be more closely confined to the Galactic plane. Almost all UC HII regions in our Galaxy lie within $2^{\circ}$ of the Galactic plane, and the overwhelming majority lie within $1^{\circ}$ of the Galactic plane (Churchwell 1990). Planetary nebulae have a much wider distributionin Galactic latitude, reflecting their mixed Population I and Population II origins, butover $15 \%$ of catalogued planetary nebulae are within $2^{\circ}$ of the Galactic plane (from Perek and Kohoutek 1965). M1-78 is a Galactic plane object, having a latitude of $b^{I I}=1^{\circ} 28^{\prime} 29^{\prime \prime}$. This would argue weakly that it is more likely to be an UC HII region than a PN, but it is obviously inconclusive. 
Ultracompact HII regions are often less regular in appearance than planetary nebulae, which are usually either spheroidal shells or bilobate. In contrast UC HIIregions show a variety of morphologies, including a significant percentage of irregular and"cometary" shapes. Only 4\% of compact HII regions have "shell-type" morphologies — theclassical PNe morphology consisting of a dense outer shell surrounding a central hollow (Woodand Churchwell 1989). There are however many UC HII regions that are either spheroidal or unresolved $(\approx 60 \%$ of examples, Wood and Churchwell 1989), and there are also a fewdistinctly irregular planetary nebulae (e.g. NGC7027). The morphology of M1-78 has been determined by Scott and Harris (1978) who find that the nebula has bipolar bright region with asize of $4.0 " \times 1.8$ " aligned along a north-west to south-east line, as would be fairly typical ofa small planetary nebula. However, they also find an irregular fainter region extending some 4"to the north-east, which would seem more typical of an UC HII region. Scott and Harris find that neither classification can be ruled out based on the observed morphology.

Ultracompact HII regions generally occur in or near active starforming regions, and consequently are often seen in groups. The stellar progenitors of PNeare aged stars that have wandered far from their natal clouds, and therefore are usuallyisolated or lie within aged clusters. A radio survey of the region surrounding M1-78 by Scott and Harris (1978) does not show any nearby HII regions that can clearly be associated with M1-78. This would argue weakly that the nebula has probably left its formation region and istherefore likely to be a PN. But alternatively, M1-78 may simply be the only massive star to form in that particularcloud.

Ultracompact HII regions have fairly uniform chemical compositionsreflecting the chemical composition of the general interstellar medium (hereafter ISM).Planetary nebulae often have significantly modified chemical compositions as a result of theconvective mixing of nuclearprocessed material into the outer layers of their precursor stars.Zijlstra et al. (1990) note that the chemical composition of M1-78 is enriched in nitrogen with respect to oxygen when compared to typical HII regions, although no firm numbers are given.Perinotto (1991) gives the nitrogen to oxygen abundance ratio of 0.47 for M1-78 - near the average value for his PNe but over twice the canonical N:O abundance ratio of 0.22 for theISM (Mezger 1972). This significant deviation from the chemical composition of the ISMwould favour identification of M1-78 as an evolved object such as a PN.

Ultracompact HII regions often have lower expansion velocities thanplanetary nebulae. Typically, compact HII regions have ionized-gas expansion velocityless than or equal to the sound speed in their gas, that is $v_{\text {exp }} \lesssim 10 \mathrm{~km} \mathrm{~s}^{-1}$ Exceptions to this occur when the ionizing star itself has a supersonic velocity of $>10 \mathrm{~km} \mathrm{~s}^{-1}$ with respect to its natal nebula, as occurs in multiple-star OB associations. In such cases, bow shocks develop thatdistort the HII region into a cometary structure (Mac Low et al. 1991). Planetary nebulae as a group have somewhat 
higher measured ionized-gas expansion velocities, averaging $v_{\text {exp }} \approx 20 \mathrm{kms}^{-1}$ (Weinberger 1989), and as such typically expand supersonically, even those with a regular shell-type morphologies. The measured expansion velocity of M1-78 is $v_{\text {exp }}=(25.3 \pm 2.0) \mathrm{km} \mathrm{s}^{-1}$ (Gussie and Taylor 1989). This supersonic expansion velocityfavours classification as a PN since the morphology of the nebula is not cometary, and displays noother manifestations of a bow shock created by a supersonic central star.

The active star forming regions that compact HII regions aregenerally associated with clouds of molecular gas which are observable as CO emission (e.g. Churchwell et al. 1992). In contrast, the majority of planetary nebulae show a surprising paucity ofCO emission, although some are known to be relatively weak CO sources (e.g. Huggins and Healy 1989). M1-78 is a powerful source of $\mathrm{CO}$ emission, and the only known $\mathrm{PN}$ with acomparable intensity of $\mathrm{CO}$ emission is NGC7027. There are however considerable differences between the COclouds of NGC7027 and M1-78. Zuckerman et al. (1977) report an extended distribution for the CO of M1-78, while the CO emission of NGC7027 is confined to within one arc minute of the center of the ionized nebula (Phillips et al. 1991). Bujarrabal et al. (1988) report that the linear diameters of $\mathrm{CO}$ clouds of various young planetary nebulae andproto-planetary nebulae is $0.23 \mathrm{pc}$, to within a factor of 2 . A linear size of $0.23 \mathrm{pc}$ forthe CO cloud of M 1-78 would require the maximum distance to M1-78 be an improbably low $260 \mathrm{pc}$. This would imply that the $\mathrm{CO}$ in M1-78 is associated with an external molecular cloud, and so aclassification of the nebulae as an UC HII region would be supported.

The CO emission lines M1-78 also have a very narrow bright component and a broader low-intensity component (figure 2), while the CO emission lines ofplanetary nebulae are typically parabolic, flat-topped, or double-peaked in shape (e.g. Huggins and Healy 1989). Furthermore, the systemic velocities of the CO lines of NGC7027 closely match the velocity of its optical emission lines, while the velocities of the molecular andoptical emission lines are displaced by $\approx 10 \mathrm{~km} \mathrm{~s}^{-1}$ in the case of M1-78. This would favour identification as an UC HII region because the molecular emission lines of UC HII regions are often shifted with respect to the optical emission lines by a similar velocity (Forster et al. 1990).

An alternative explanation for the strange $\mathrm{CO}$ emission properties of M1-78 is that it may be a planetary nebula that possesses a $\mathrm{CO}$ maser in its molecular envelope.Spherically-symmetric radiative transfer models of the expanding neutral envelopes of AGBstars by Morris (1980) have shown that under certain fairly narrowly defined conditions ofmass-loss rate and expansion velocity, $\mathrm{CO}$ maser emission may occur. Morris identified theprotoplanetary nebula CIT6 as undergoing possible CO maser emission because it has displayedchanges in the shape and intensity of its $J=1 \rightarrow 0 \mathrm{CO}$ emission line over a period of about two years, which Morris attributes to the switching off of a weak CO maser. Anobvious objection to the 
hypothesis that M1-78 has a $\mathrm{CO}$ maser is that the $\mathrm{CO}$ maser emission in the Morris' modelsare double-peaked while M1-78 displays only a single red shifted peak. However, theMorris' models are spherically symmetric and therefore have identical conditionsin both the near (blue shifted) and far (red shifted) sides of their envelopes. It isplausible that the lop-sided CO emission observed in M1-78 is the result of an asymmetric mass distribution. The apparent rapid shut off of the $\mathrm{CO}$ maser in CIT6 illustrates how sensitive $\mathrm{CO}$ maser emission is to density and temperature changes, making it reasonable that $\mathrm{CO}$ maseremission could exist in only one side of a circumnebular envelope. This hypothesis couldhowever not easily explain the velocity of the observed HI absorption line (Gussie and Taylor 1994), which is identical to that of $\mathrm{CO}$ emission. The presence of HI absorption would indicate that the bulk of the red shifted neutral gas is between us and the ionized nebula, and musttherefore be either unrelated to the ionized nebula or undergoing infall rather than the outflowexpected of a PN molecular shell. The CO emission of M1-78 would therefore tend to favour a classification as an UC HII region.

The luminosity of the nebula would also tend to argue against itsidentification as a PN. Based on far-infrared data taken by the Infrared Astronomy Satellite,Puche et al. (1988) find a total far-infrared luminosity of $L_{*}=\frac{D}{6 \mathrm{kpc}} 10^{5} L_{\odot}$, where $D$ is the nebula's distance in kiloparsecs. If the range in planetary nebula nucleus masses is takento be $0.5 M_{\odot}$ to $0.8 M_{\odot}$ (Pauldrach et al. 1988), then the theoretical upper limit to the luminosity of PNe is $\approx 20000 L_{\odot}$ based on models of Paczynski (1970) and Kippenhahn (1981). Anupper limit to the distance of M1-78 would be $D=1.2 \mathrm{kpc}$ if it were a PN. However, M1-78 suffers a total extinction of $\mathrm{A}_{\mathrm{H} \beta}=10.2$ magnitudes (Scott and Harris 1978), while Allen(1973) list the mean absorption of optical radiation in the Galactic plane as $\mathrm{A}=1.9$ magnitudes $\mathrm{kpc}^{-1}$. M1-78 and its surrounding environs must therefore provide an additional opticalextinction of $\mathrm{A}_{\mathrm{H} \beta}=8.1$ magnitudes if the nebula were a PN at $1.2 \mathrm{kpc}$ distance. This is veryhigh, but not entirely unprecedented. Woodward et al. (1992) have shown that the optical extinction of NGC7027 (while very patchy) reaches $\mathrm{A} \approx 8$ magnitudes at least at some points on the face of the nebula's ionized region. Various proto-planetary nebulae also showhigh optical extinctions (e.g. Soptka et al. 1985). If M1-78 were a PN it would then seem to be a very young, massive, and dusty object, perhaps the most massive example known.Its properties would perhaps place it as an intermediate object in evolutionary termsbetween the massive PN NGC7027 and the proto-planetary nebula CRL618, which possesses a massive and dusty molecular envelope (Bunjarrabal et al. 1988) but not as yet as highly a developed ionised region as M1-78. Alternatively, the nebula may be a more ordinary PN that happensto lie behind a dense molecular cloud; at least a few such juxtapositionsmust exist. However compact HII regions are typically located near (or within) cold molecular clouds and often suffer from tremendous optical extinction. In fact, most UC HII regions are completely obscured at optical wavelengths and are only seen as radio and infraredsources. 
Ultracompact HII region regions are young objects, and as such they are assumed tohave much smaller peculiar velocities than do $\mathrm{PNe}$; that is, their motionis expected to follow the Galactic rotation curve more closely. Kinematic distances aretherefore routinely used to estimate the distances to UC HII regions, but they have long since been proven to be unreliable in determining distances to PNe. Therefore, if M1-78 were shown to have a distance significantly different from its kinematic value, identification as a PNwould be indicated. The systemic velocity of the ionized nebula of M1-78 (Gussie and Taylor 1989) is found to be $v_{L S R}=(-76 \pm 2) \mathrm{kms}^{-1}$ as measured by optical spectroscopic observations of the [OIII] lines. The optical velocity is supported by measurements of hydrogen radiorecombination lines by Terzian et al. (1984) and Churchwell, Terzian, and Walmsley (1976). This wouldimply a kinematic distance to the nebula of $D \approx 8 \mathrm{kpc}$.

Comparison of the kinematic distance with the actual distance is howeverdifficult, since the distance to M1-78 is poorly known. Various statistical PN distance determinationmethods have given distances from $3 \mathrm{kpc}$ to $5 \mathrm{kpc}$ (Cahn and Kaler 1971,Acker 1978, Maciel 1984), but the reliability of these methods has not been shown and is inconsiderable doubt; and they would of course be inapplicable anyway if M1-78 were not a PN.

More reliable attempts to determine the distance to M1-78 include that by Puche et al. (1988), who present $\lambda=21 \mathrm{~cm}$ spectral line observations which show HI absorption features at $v_{L S R}=-10 \mathrm{~km} \mathrm{~s}^{-1}, v_{L S R}=-45 \mathrm{~km} \mathrm{~s}^{-1}$, and $v_{L S R}=-65 \mathrm{~km} \mathrm{~s}^{-1}$. Puche et al. identify the $v_{L S R}=-10 \mathrm{~km} \mathrm{~s}^{-1}$ absorption feature as interstellar absorption caused by HI the Local Spiral Arm and the $v_{L S R}=-45 \mathrm{~km} \mathrm{~s}^{-1}$ absorption feature as interstellar absorption caused by HI in the Perseus Arm. The $v_{L S R}=-65 \mathrm{kms}^{-1}$ absorption feature is tentatively identified as being due to a third spiral arm beyond the Perseus Arm. The presence of the HI absorption would require the nebula to be between $6 \mathrm{kpc}$ and $8 \mathrm{kpc}$, in accord with the kinematicdistance. This distance would then preclude the possibility that M1-78 is a planetary nebula on the basis of its impossibly high required luminosity. This distance would also placethe nebula $\approx 200 \mathrm{pc}$ above the Galactic plane, which is an unusually large, but not animpossibly large, height for an UC HII region.

The distance might be determined by further high-resolution radiocontinuum observations. If the nebula were a PN (and therefore at a distance of $D=1.2 \mathrm{kpc}$ ) then the rate of angular growth of the ionized region would be $\frac{\Delta \theta}{\Delta \mathrm{t}}=0.0045^{\mathrm{N}} \mathrm{yr}^{-1}$. A high-resolution $\lambda=2 \mathrm{~cm}$ continuum image of the nebula taken at the present epoch couldtherefore be used to measure the apparent expansion by comparison with the image taken by Scott andHarris in 1978.

The uncertainty regarding the nebula's luminosity could be answeredif its central star (or stars) was observed. However, the author knows of no observationsor detections of the 
nebula's central star, and the large external and internal dustextinction makes it unlikely that the star's optical emission would be detectable.

4) Conclusions

It is clear that the various distinguishing characteristics ofplanetary nebulae and ultracompact HII regions do not give an unambiguous classification for M1-78. On the whole, a classification as an UC HII region would seem to be favoured but the unusual chemical composition of the nebula and its high expansion velocity wouldpresent serious problems for such a classification. I consequently favour a rather uniqueclassification for this object: an UC HII region with a post-main sequence central star.

Wood and Churchwell (1990) have shown that UC HII regions can not be transitory phenomena that are created at the start of main sequence evolution as waspreviously assumed, but must instead persist for much of a massive star's mainsequence life time. They propose and examine many possible mechanisms for the preservation of anidentifiable UC $\mathrm{H}$ II region long past the time that a freely expanding UC HII region would require to disperse into the ISM. If an UC HII region persisted for even longer time scales than those suggested byWood and Churchwell, then a very aged UC HII region would receive chemical enrichment from a post-main sequence stellar wind. Such a wind would releasenuclear-processed stellar material brought to the photosphere by convection during one or more "dredge-up" phases of stellar evolution. Such deep convective mixing is not expected until aftermain sequence evolution (Greggio 1983). The observed over-abundance of nitrogen in M1-78 would suggest that the central star is (or was) a nitrogen-rich Wolf-Rayet star (spectralclass WN). The evolutionary status of WN stars has been a matter of some debate (e.g. Underhill 1983), but they are now generally regarded as post-main sequence objects with a high originalmass (e.g. Abbott and Conti 1987).

As a point of clarification, the central stars of several PNe areknown to have Wolf-Rayet type stellar spectra (e.g. NGC6369). It is however believed because of the differences in the distributions of $\mathrm{PNe}$ and $\mathrm{OB}$ associations that these "Wolf-Rayet"stars have quite different origins than those associated with young O stars, and are insteadpost AGB objects of much more moderate original mass. These PN Wolf-Rayet stars are not consideredhere. Instead, it is suggested that the power source of M1-78 is a "classical" Wolf-Rayet star, and as such is an extreme Population I object.

The high luminosity of the nebula is not a difficulty if a WN star wereits power source, as the absolute magnitudes of such objects are as high as $M_{v}=-8$ (Abbott and Conti 1987). WN stars are also often seen to have close O-type binary companions(Roberts 1962), increasing the available luminosity even further. 
It is known that WN stars are usually significantly less massivethan their O star companions (Massey 1981), despite being the (apparently) moreevolved of the pair. This is easily explained if much of the stellar mass is lost to the Wolf-Rayetstellar wind. Mass loss in Wolf-Rayet stars is observed at the $\approx 5 \times 10^{-5} M_{\odot}$ year-1 rate (Abbott et al. 1986), which over the expected Wolf-Rayet lifetime of $\approx 4 \times 10^{5} \mathrm{yr}$ (Chiosi et al. 1978) would strip $\gtrsim 10 \mathrm{M} \odot$ from the star. Wolf-Rayet stars are therefore regarded as the barecores left by the post-main sequence loss of the outer envelope of an O star (Maeder 1983).So-called "Ring Nebulae" have been observed around many Wolf-Rayet stars which are believed toconsist of the remnant wind material augmented by swept-up interstellar matter. Chemicalcompositions of the nebulae around WN stars have shown nitrogen to helium abundance enhancementsof 3 to 10 times over that of the general ISM (Kwitter 1981, Kwitter 1984, Esteban et al. 1990). The observed by nitrogen abundance anomaly in M1-78 is therefore easily accounted for by the addition of a nuclear-processed stellar wind material to a pre-existing UC HII region.

WN star mass loss could also explain the supersonic expansionobserved in M 1-78. Terminal wind velocities in Wolf-Rayet stars are observed to be as highas $3700 \mathrm{~km} \mathrm{~s}^{-1}$ (Willis 1982), making the kinetic energy of Wolf-Rayet stellar winds asignificant proportion of the stellar energy budget; perhaps as high as $10 \%$. Such a wind would\%\%impact significantly on the kinematics of the surrounding gas. Kwok et al. (1978) have proposed an "interacting stellar winds" model for planetary nebula evolution, where a fast $\left(\gtrsim 2000 \mathrm{~km} \mathrm{~s}^{-1}\right)$ wind from the PN central star overtakes the slower, relatively dense wind of AGB mass loss. This accelerates the ionised shell. Applying this same idea to an UC HII region with a WN central star, the star's fast wind would accelerate the gas of the natal molecular cloud,rather than material from an earlier mass-loss episode as in the case in PN evolution.

\section{5) Summary}

M1-78's infrared colours, its isolation, its chemical composition, andits expansion velocity would argue that the nebula is a young but massive planetary nebula.However the molecular emission would favour a classification as an ultracompact HII region, and the nebula's luminosity makes M1-78's classification as a planetary nebula untenable if the distanceestimate of Puche et al. (1988) is accepted. The contradictory nature of the evidenceconcerning the classification of M1-78 may be resolved if the nebula were an ultracompact HII region that has undergone significant chemical alteration by the stellar wind of apost-main sequence central star. The nebula's luminosity and enriched nitrogen abundance wouldargue that the central star is (or was) of spectral class WN. Observable ultracompact HIIregions would then necessarily persist beyond the entire main sequence lifetime of a massive star. 
6) Acknowledgments

This research was supported by a grant from the Australian ResearchCouncil. This research has made use of the SIMBAD database, operated by CDS,Strasbourg, France. 


\section{Literature Cited}

Abbott, D. C., Bieging, J. H., Churchwell, E., andTorres, A. V. 1986, ApJ, 303, 239.

Abbott, D. C., and Cont, P. S. 1987, ARA\&A, 25, 113.

Antonopolou, E., and Pottasch, S. R. 1987, A\&A, 173, 108.

Allen, C. W. 1976, Astrophysical Quantities, (London: Athlone), p 263.

Bujarrabal, V., Gómez-González, J.,Bachiller, R., and Martín-Pintado, J. 1988, A\&A, 204242.

Cahn, J. H., and Kaler, J. B. 1971, ApJS, 22, 319.

Chiosi, C., Nasi, E., and Sreenivasan, S. 1978 A\&A, 63, 103.

Churchwell, E., 1990 A\&AR, 2, 279.

Churchwell, E., Terzian, Y., and Walmsley, M. 1976, A\&A, 48, 331.

Churchwell, E., Walmsley, C.M., and Wood, D.O.S. 1992, A\&A, 253, 541.

Esteban, C., Vilchez, J. M., and Machado, M. A. 1990, A\&A, 227, 515.

Forster, J. R., Caswell, J. L., Okumura, S. K.,Hasegawa, T., and Ishguro, M. 1990, $A \& A, 231,473$.

Greggio, L. 1984, IAU Symp, 105, 299, 329.

Gussie, G. T., and Taylor, A. R. 1989, PASP, 101, 873.

Gussie, G. T., and Taylor, A. R. 1994, MNRAS, submitted.

Huggins, P. J., and Healy, A. P. 1989, ApJ, 346, 201.

Kippenhahn, R. 1981, $A \& A, 102,293$.

Kwok, S., Purton, C. R., and Fitzgerald, M. P. 1978, ApJ, 219, L125.

Kwitter, K. B. 1981, ApJ, 245, 154.

Kwitter, K. B. 1984, ApJ, 287, 840.

Mac Low, M., Van Buren, D., Wood, D. O. S., andChurchwell, E. 1991, ApJ, 369, 395. 
Massey, P. 1981, ApJ, 246, 153.

Maciel, W. J. 1984, A\&AS, 55, 253.

Maeder, A. 1984, IAU Symp, 105, 299.

Mezger, P. G. 1972, in Interstellar Matter, ed. Wickramasinghe, N. C., Kahn, F. D., and Mezger, P. G., (Astronomical Institute: University ofBasel), p 6.

Morris, M. 1980, ApJ, 236, 823.

Pauldrach, A., Kudritzki, R. P., Gabler, R., andWagner, A. 1988, in Mass Loss Outflows from Stars and Galactic Nuclei, eds. L. Bianchi and R. Gillmozzi, (Dordrecht:Kluwer), p 63.

Paczynski, B. 1970, Acta.A, 20, 47.

Perek, L., and Kohoutek, L. 1967, Catalog of Planetary Nebulae, Prague: Czechoslovak Institute of Sciences.

Perinotto, M. 1991, ApJS, 76, 687.

Phillips, J. P., Mampaso, A., Williams, P. G., andUkita, N. 1991, A\&A, 247, 156.

Pottasch, S. R., Baud, B., Beintma, D.,Emerson, J., Habing, H. J., Harris, S., Houck, J., Jennings, R., and Marsden, P. 1984, A\&A 138, 10.

Puche, D., Zijlstra, A. A., Boettcher, C.,Plante, R. L., Wilcots, E. M., Wilkin, F. P., Krause, S., Sergo, S. P., Bierman, G. S., Ge, J.,Holliman, J. H., Wu, X., and Zhao, J. H. 1988, A\&A, 206, 89.

Roberts, M. S. 1962, AJ, 67, 79.

Soptka, R. J., Hildebrand, R., Jaffe, D. T.,Gatley, I., Roellig T., Werner, M., Jura, M., and Zuckerman, B. 1985, ApJ, 294, 242.

Terzian, Y., Higgs, L. A., MacLeod, J. M., andDoherty, L. H. 1974, AJ, 79, 1018.

Underhill, A. B. 1983, ApJ, 265, 933.

Willis, A. J. 1982, MNRAS, 198, 897.

Weinberger, R. 1989, A\&AS, 78, 301. 
Wood, D. O. S., and Churchwell, E. 1989, ApJS, 69, 831.

Zijlstra, A. A., Pottasch, S. R., Bignell. C. 1990, $A \& A S$, 82, 273.

Zuckerman, B., Palmer, P., Morris, M., Turner, B. E.,Gilra, D. P., Bowers, P. F., and Gilmore, W. 1977, ApJ, 211, L97. 


\section{Figure Captions}

\section{1) IRAS Colour-Colour Plot of Planetary Nebulae and Compact HIIRegions}

IRAS colour-colour plot of a sample of planetary nebulae (circles)and compact HII regions (squares) as well as M1-78 (cross) showing flux at $\lambda=12 \mu \mathrm{m}$ over flux at $\lambda=25 \mu \mathrm{m}$ in Jansky as a function of flux at $\lambda=25 \mu \mathrm{m}$ over flux at $\lambda=60 \mu \mathrm{m}$, also in Jansky. Planetary nebulae and compact HII regions occupy distinct regions on this plot,although some overlap occurs. M1-78 has a position intermediate between the two classes of objects, but is perhaps more similar to the planetary nebulae. The planetary nebular dataare from Pottasch et al. (1984) and consist of 41 bright planetary nebulae. The compact HIIregion data are from Antonopolou and Pottasch (1987) and consist of 64 nebulae.

\section{2) $J=3 \rightarrow 2$ CO Spectrum of M1-78}

The $J=3 \rightarrow 2$ CO spectrum of M1-78 from Gussie and Taylor (1994). The local standard of rest velocity of the ionized nebula is shown by the arrow. The moleculargas is red-shifted with respect to the ionized gas, and displays a highly-peaked line shape thatis atypical of planetary nebulae. Note the highly red-shifted component near $-7 \mathrm{kms}^{-1}$. 
Figure 1

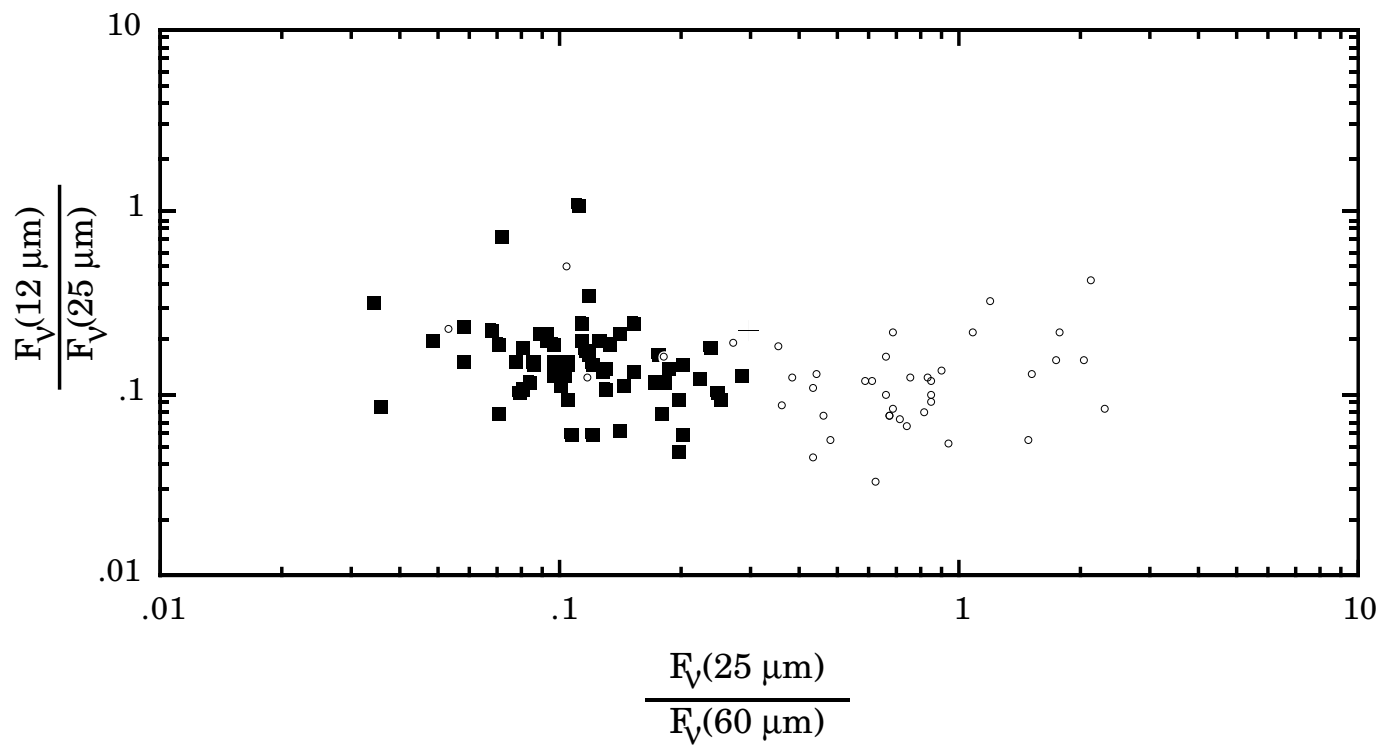


Figure 2

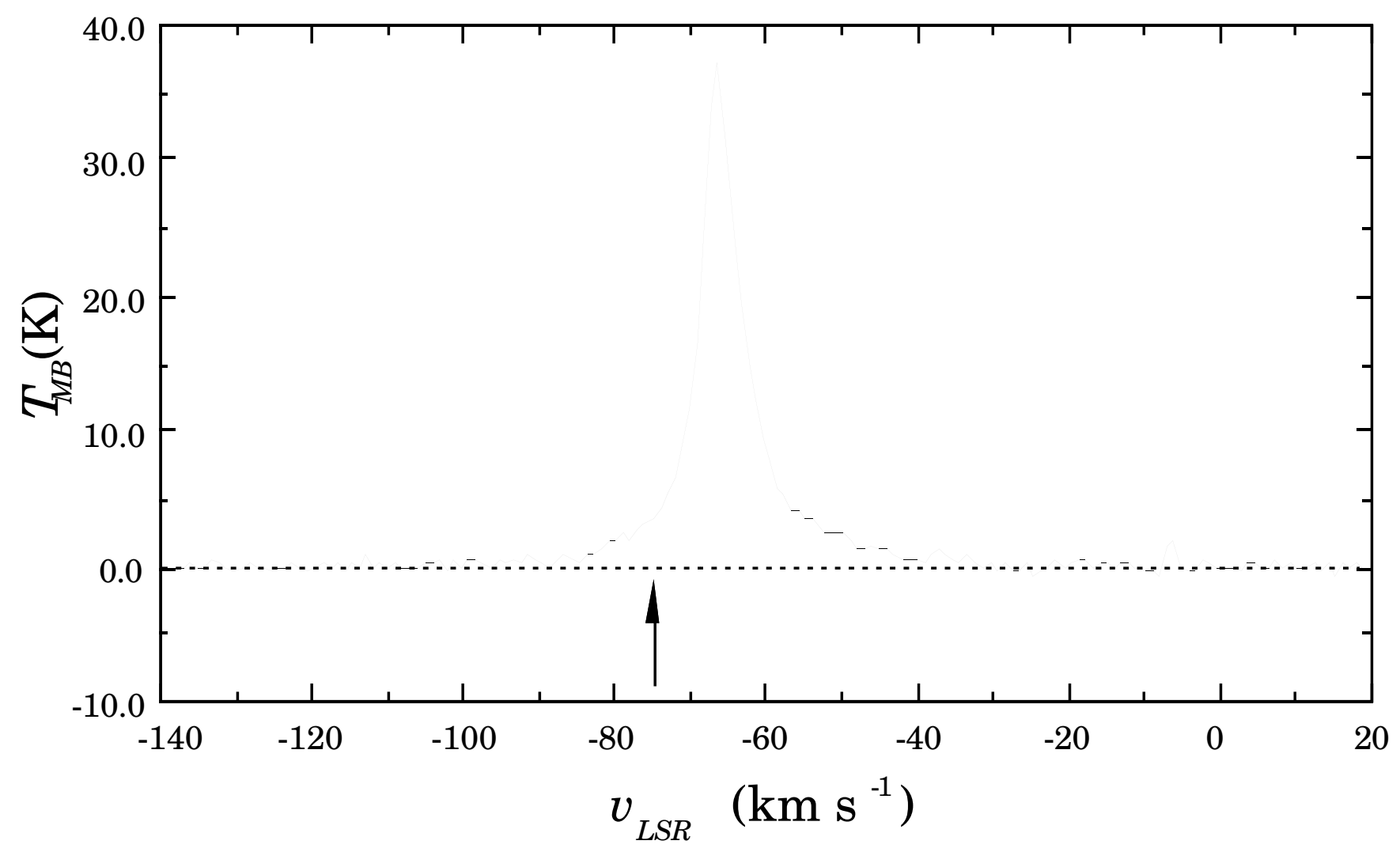

